\title{
Marquage casuel des dépendants nominaux du verbe en ancien français: approche dépendancielle
}

\author{
Mazziotta, Nicolas \\ Universität Stuttgart \\ nicolas.mazziotta@ulg.ac.be
}

\section{Introduction}

Cette contribution ${ }^{1}$, qui adopte un point de vue résolument dépendanciel, traite de la syntaxe de la proposition en ancien français (désormais, « afr. »). Nous examinons la manière dont les actants nominaux les plus proches du verbe sont identifiés par des morphèmes spécifiques.

Il a été clairement démontré que le système casuel de l'ancien français n'était pas suffisamment fiable pour permettre le discernement entre les différents types de dépendants du verbe ${ }^{2}$. En particulier, les études ont montré :

1. que la valence du verbe et les propriétés sémantiques des actants sont plus importantes que la déclinaison pour déterminer quel actant est le sujet et quel actant est l'objet (Schøsler, 1984);

2. que les paradigmes de déclinaison sont si hétérogènes qu'il est impossible de les décrire de manière systématique (Chambon, 2003; Chambon et Davidsdottir, 2007).

S'appuyant sur cette instabilité, certains n'hésitent pas à affirmer que le système casuel a avant tout une valeur sociolinguistique : celui qui le maîtrise démontre principalement qu'il est bien intégré à la communauté linguistique (Detges, 2009, en partic. 117). Les descriptions grammaticales générales s'accordent avec ces conclusions, mais continuent à présenter un système casuel sous la forme d'une liste de paradigmes et d'exceptions (Moignet, 1988; Buridant, 2000). Cela est bien compréhensible : bien que peu fiables, les marques actancielles restent observables et leur emploi n'est pas complètement anarchique. Les règles qui gouvernent leur emploi sont intégrées au reste de la grammaire, que le locuteur mobilise dans son ensemble à chaque acte de langage (Schøsler, 2013, 173-175). Il appert que ces règles contraignent l'apparition des marques, si bien que ces dernières ne peuvent apparaitre dans n'importe quel contexte syntaxique.

Nous allons tenter de modéliser les relations entre les mots et les morphèmes impliqués dans les relations actancielles traditionnellement nommées « sujet » et « objet direct », c'est-à-dire celles qui impliquent les termes nominaux dépendant du verbe dans les constructions strictement intransitives et transitives directes. Il apparaîtra clairement ci-dessous que la hiérarchie des segments n'est pas aisée à déterminer et que même les exemples les plus simples révèlent la complexité de la structuration interne du syntagme nominal.

Une large place est accordée ici à la dimension théorique et formelle. Nous voudrions ainsi favoriser les échanges entre la linguistique de l'afr. et la linguistique générale, en particulier les modèles dépendanciels. En effet, comme l'expliquaient déjà Peter Stein et Claudia Benneckenstein en 2006, très peu d'aspects de cette langue ont été traités à l'aide d'un modèle dépendanciel ${ }^{3}$.

Nous envisageons la progression de notre exposé comme un dialogue entre les principes généraux et les données. Nous aborderons tout d'abord le critère de la valence passive $e^{4}$, proposé par la Théorie Sens-Texte pour départir le gouverneur du subordonné dans une relation de dépendance (sec. 2). Nous poursuivrons en exploitant l'analyse dépendancielle des relations unissant les formants morphologiques du mot, proposée par Thomas Groß dans un cadre dépendanciel (sec. 3). Nous aurons ensuite besoin des concepts connexes de spécification (sec. 4) et de cumul de marques (sec. 5) selon Alain Lemaréchal, qui décrit la manière dont les relations syntaxiques sont précisées par la présence parfois facultative de marques grammaticales qui se combinent. Ces discussions nous mèneront enfin à revoir les définitions et les principes qui opposent la dépendance morphologique à la dépendance syntaxique dans la Théorie Sens-Texte (sec. 6). 
Une autre conséquence de la relativement pauvre interaction entre la linguistique dépendancielle et la description de l'ancien français est que la constitution de corpus exploitant ces formalisations est très récente (cf. le projet Syntactic Reference Corpus of Medieval French «SRCMF », Prévost et Stein 2013). Cette étude repose sur l'examen du texte La queste del saint Graal, dont nous retirons les observations qui illustreront notre $\operatorname{propos}^{5}$. Nous nous sommes servi des annotations syntaxiques fournies par le projet SRCMF, qui portent sur le texte de la nouvelle édition de Christiane Marchello-Nizia (Marchello-Nizia et Lavrentiev, 2013), mais nous proposons une autre formalisation que celle que livre la ressource. Tous les exemples cités sont tirés de cette édition.

\section{Critère de la valence passive}

Soit les deux formes $f_{1}$ et $f_{2}$, liées par une relation de dépendance, laquelle des deux formes doit-elle être considérée comme le gouverneur de la relation?

\subsection{Définitions et principes d'analyse}

Cette question a sa pertinence quelle que soit la nature du modèle syntaxique employé (qu'il soit syntagmatique ou dépendanciel). De nombreux critères ont été cités depuis l'article d'Arnold Zwicky (1985), mais il nous semble que c'est Igor Mel'čuk (2009) qui a livré la liste de critères la plus solide jusqu'à présent. Selon la Théorie Sens-Texte (désormais «TST») telle que cet auteur la définit, l'orientation d'une relation entre $f_{1}$ et $f_{2}$ est prioritairement déterminée par le critère syntaxique de la valence passive du syntagme ${ }^{6}$ :

La valence passive d'un lexème ou d'un syntagme est l'ensemble des fonctions syntaxiques que le lexème ou le syntagme peut assumer dans une construction plus étendue (éventuellement après avoir subi des modifications dans sa désinence). En d'autres termes, la valence passive d'un lexème ou d'un syntagme est sa distribution syntaxique. (Mel'čuk, 2009, 4, nous traduisons)

Le gouverneur d'une relation syntaxique contrôle la valence passive de la construction qu'il forme avec ses dépendants ; c'est-à-dire que $f_{1}$ gouverne $f_{2}$ si la distribution de la construction $f_{1}+f_{2}$ est davantage celle de $f_{1}$ que celle de $f_{2}$. Il est important de noter que le critère concerne la distribution syntaxique, sans référence à l'ordre des mots et des structures.

(1) bel comencement lor a Diex mostré $(257,22)$

Dans l'ex. 1, le mot comencement gouverne le mot bel (comencement $\rightarrow$ bel), parce que la distribution de bel comencement est plus celle de comencement que celle de bel $^{7}$.

D'autre part, selon Igor Mel'čuk (2009, 23-24), il n'existe que quatre moyens linguistiques d'exprimer un contenu :

- les lexèmes, envisagés comme des mots libres non fléchis (sans distinction entre les mots « lexicaux »et les mots « grammaticaux »);

- l'ordre des lexèmes ;

- la prosodie ;

- la flexion.

À l'exception de la flexion, toute phrase, quelle que soit la langue considérée, mobilise ces moyens simultanément pour exprimer soit un contenu sémantique, soit un contenu syntaxique (c'est-à-dire les relations entre les mots). Pour la TST, les lexèmes sont organisés au niveau de la structure syntaxique de surface, mais les autres moyens d'expression du contenu ne font pas partie de ce niveau de structuration, bien qu'ils permettent de le reconstruire. Ainsi, la distinction entre le sujet et l'objet en français moderne est exprimée par l'ordre des mots SVO, mais cet ordre ne fait pas partie de la structure, qui reste organisée sous la forme 
d'un arbre projectif. Si elle est avérée, la correspondance entre l'ordre linaire et l'ordre représenté dans la structure syntaxique est fortuite. De même, la flexion n'est qu'un indice de la structure.

\subsection{Application à l'ancien français}

L'application du critère de la valence passive couplée à ce rejet de l'analyse syntaxique des marqueurs liés engendre une hiérarchisation homogène des dépendances à l'œuvre dans le syntagme nominal dépendant du verbe en afr. En effet,

- si nul cas n'est fait de la désinence des mots, reléguée au niveau morphologique, la forme du nom et celle de l'article ne contraignent jamais la distribution du syntagme;

- le caractère non obligatoire de l'article, s'il n'est pas décisif en soi, renforce l'idée que c'est bien le nom qui détermine la valence passive.

En conséquence, l'article se trouve systématiquement en position subordonnée par rapport au nom. L'analyse du sujet et de l'objet de l'ex. 2 est ainsi celle de la fig. 1. Pour ce schéma et les suivants, les types de dépendances sont distingués par les étiquettes placées sur les flèches lorsque nous l'avons jugé utile. Le jeu d'étiquettes, qui correspond aux fonctions classiques, ne devrait pas poser de problème de compréhension ; il n'est pas discuté ici.

(2) li chevaliers conduit le cheval [...] $(149,10)$

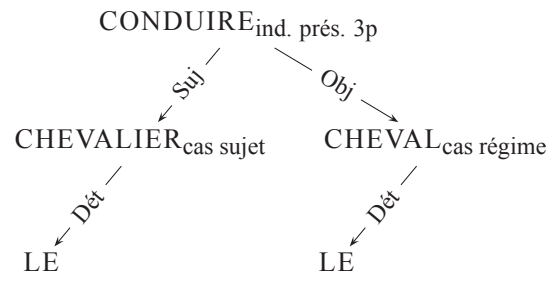

Fig. 1 - Analyse de li chevaliers conduit le cheval selon la TST

Il apparaît clairement que placer les catégories grammaticales en indice de chaque lexème ${ }^{8}$ a une incidence majeure sur l'arbre : ces informations ne font pas partie de la hiérarchie.

\section{Analyse syntaxique des formants du mot}

Le critère de la valence passive a été pensé dans le cadre de l'analyse des relations qui unissent les mots et non les morphèmes/morphes. Qu'en est-il de son application à ces unités ?

\subsection{Hiérarchie des morphes}

Si l'on accepte de décrire syntaxiquement la structure interne des mots et d'étendre en conséquence le modèle de la TST à une analyse des relations syntagmatiques entre les différents formants, il est possible de rendre compte du potentiel syntaxique des morphes. C'est ce que fait Thomas Groß (2011) dans un cadre dépendanciel ${ }^{9}$. S'agissant d'une représentation de surface, ce ne sont pas les morphèmes (unités abstraites), mais bien leur réalisation, les morphes, qui sont pris en considération.

Thomas Groß propose de traiter les morphes liés ${ }^{10}$ de la même manière que les mots, c'est-à-dire de les représenter de manière disjointe de leur base lexicale dans l'arbre syntaxique. L'idée est appliquée depuis longtemps dans les grammaires syntagmatiques : on la trouve déjà dans la définition fondamentale des 
constituants immédiats chez Leonard Bloomfield (1933, ch. 10), qui considère que les constituants immédiats peuvent être des morphèmes liés ou libres. Par ailleurs, l'analyse faisant de la désinence une tête fonctionnelle est très répandue au sein de la communauté de linguistes utilisant la théorie du gouvernement et du liage (Haegemann, 1994, en partic. ch. 11). Cette conception est aussi très proche de la notion de tripartition de la syntaxe (Lazard, 1984). Gilbert Lazard distingue trois niveaux : le niveau de la proposition, celui du syntagme et le niveau interne au mot. Ce dernier niveau est traditionnellement nommé morphologie, mais ce terme classique mène à considérer le mot comme une chaîne monodimensionnelle, alors que la syntaxe correspond à une structure bidimensionnelle (au moins). Il nous semble que cette idée est également déjà clairement exprimée par Paul Garde lorsqu'il définit le concept de dépendance en ces termes (1981, 159, nous soulignons) :

[T]oute relation syntaxique (entre morphèmes dans le mot, entre mots dans la phrase) s'établit entre deux termes dont l'un, subordonné, dépend de l'autre, principal. Le terme principal (s'il s'agit de mots, le mot principal) est celui qui contient l'information sur les rapports syntaxiques entre l'ensemble constitué par le principal et son subordonné et l'environnement de cet ensemble.

Néanmoins, cette idée a été proprement ignorée dans un cadre dépendanciel, si bien que sa définition formelle n'a pas retenu l'attention.

L'argument principal qui autorise Thomas Groß à considérer les morphes liés de la sorte est leur similitude fonctionnelle avec les prépositions et les conjonctions : pour peu qu'on ne les réduise pas à un concept abstrait (le morphème), les désinences contraignent la distribution du mot qu'elles servent à fléchir de la même manière que les mots-outils. Il est donc possible d'appliquer aux morphes le critère de la valence passive. La conclusion de Thomas Groß est que ces morphes désinenciels sont des gouverneurs et que les arbres sont des outils graphiques qui représentent les relations entre les morphes de façon identique aux relations entre les mots, puisque la sémiotique des lignes reliant les formes demeure inchangée. Prenant un exemple allemand (ex. 3) impliquant une préposition, une marque de pluriel et un datif, l'auteur propose une analyse différente de celle de la TST, comme cela est visible dans la fig. 2.

(3) mit Kind -er -n

avec enfant PL. DAT.

«avec des enfants»

Les lignes pointillées dans les schémas expriment les dépendances internes qui unissent les formants morphologiques des mots et les traits d'union signifient que ces unités manquent d'autonomie ${ }^{11}$.

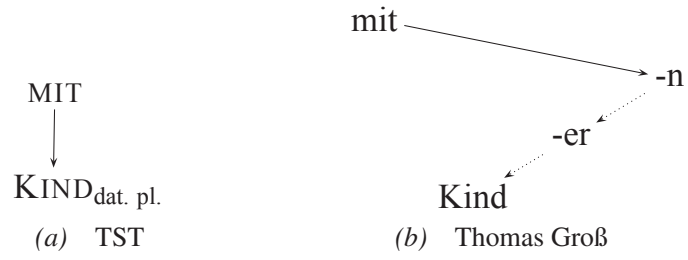

Fig. 2 - Analyse de mit Kindern selon la TST et Thomas Groß

La préposition allemande mit implique que le complément régi porte une désinence correspondant au datif, obligatoire dans ce contexte. Le dépendant de mit est n'importe quel syntagme au datif, mais uniquement un syntagme au datif (la grammaire normative considère que l'accusatif ne serait pas grammaticalement correct). De ce fait, le critère de la valence passive fait de $-n$ le gouverneur de tout le syntagme Kindern, puisque c'est ce morphe qui exprime le datif et qui contraint la distribution du syntagme. De même, la marque du pluriel -er domine la base lexicale, parce que $-n$ force l'emploi d'un mot au pluriel. 


\subsection{Application à l'ancien français}

Appliquée à l'afr., cette idée mène à disjoindre les marques casuelles du lexème qui les porte. Si le $-s$ est la marque conditionnant la fonction sujet au singulier selon le tableau classique (tab. 1), il contraint en conséquence la distribution du nom auquel il s'ajoute.

\begin{tabular}{lcc} 
& sg. & pl. \\
\hline CAS SUJET & $-\mathrm{s}$ & $\emptyset$ \\
CAS RÉGIME & $\emptyset$ & $-\mathrm{s}$
\end{tabular}

Tab. 1 - Paradigme idéal de la déclinaison en afr.

Certains féminins peuvent êtres marqués de la même manière au singulier (comme virginitez dans l'ex. 5).

(4) Josephés l'apela [...] $(33,36)$

(5) virginitez est corrompue $(120,32)$

Ainsi, dans les ex. 4 et 5 , le $-s$ de flexion (également sous sa forme $-z$ ) devient le gouverneur syntaxique du nom (même s'il en dépend d'un point de vue morphophonologique). Cette analyse est représentée dans la fig. 3 (nous laissons de côté ici les problèmes posés par l'analyse des pronoms).

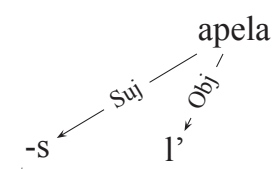

Josephé

Fig. 3 - Analyse de Josephés l'apela tenant compte des désinences

On admet généralement que n'importe quel nom peut dépendre d'un verbe, en vertu des compatibilités « naturelles » entre les parties du discours (Tesnière, 1966), mais, en termes traditionnels, la marque $-s$ sur un nom singulier implique le « cas sujet ». Cela a pour conséquence que l'éventail de fonctions syntaxiques que peut remplir le nom fléchi de la sorte est réduit (critère de la valence passive).

L'approche est séduisante, mais deux problèmes subsistent :

1. Comment traiter les « zéros » qui caractérisent traditionnellement le cas régime singulier ? Ces zéros ont-ils un sens pour les féminins en $-e$ ou pour les indéclinables?

2. Comment formaliser la description lorsque plusieurs morphes se combinent pour indiquer la fonction du syntagme, comme c'est le cas dans li chevaliers (ex. 2) ? L'analyse classique dit que le -s et l'article portent tous deux la valeur « cas sujet».

\section{Disqualification des marques zéros}

Si l'on suit Alain Lemaréchal, les marques spécifient une relation qui leur préexiste et il n'y a pas lieu de parler de «marques zéros ». Admettre la facultativité éventuelle des marques ne revient toutefois pas à dire qu'elles n'obéissent à aucune règle. 


\subsection{Spécification et zéros}

Pour expliquer comment les marques se combinent et dans quelles conditions elles peuvent éventuellement être absentes, nous ferons usage de la notion de spécification, telle que l'a définie Alain Lemaréchal dans ses travaux de linguistique générale (1997). Selon ce dernier, les unités segmentales ont reçu une attention disproportionnée de la part des linguistes. Cet écueil les a contraints à multiplier les zéros dans leurs descriptions. En toute rigueur, il convient pourtant de tenir compte de tous les procédés de marquage pour rendre compte du fonctionnement syntaxique d'une langue. Allant plus loin qu'Igor Mel'čuk (sec. 2.1), il dresse une liste hiérarchisée de ces marques, par ordre décroissant d'importance :

1. marqueurs intégratifs (prosodie et groupements);

2. ordre des lexèmes ;

3. compatibilités entre les parties du discours ;

4. marqueurs segmentaux (mots-outils libres et flexion).

Cette hiérarchie place (de manière un peu militante) les marqueurs segmentaux tout en bas de l'échelle. Selon l'auteur, ils ne sont souvent pas nécessaires pour que les unités en présence fonctionnent de concert. Les marques prosodiques et séquentielles sont toujours présentes (même si elles ne sont pas toujours faciles à interpréter) et les parties du discours entretiennent des compatibilités en langue, qui leur permet d'entrer en relation par le simple fait qu'elles sont employées ensemble - selon une approche inspirée de la théorie tesnièrienne de la connexion (Tesnière, 1966).

(6) il amerent tant Josephé [...] $(34,18)$

Ainsi, dans une construction comme celle de l'ex. 6, même si le terme Josephé ne porte aucune marque casuelle, son appartenance à la classe des noms lui permet de fonctionner comme l'objet du terme amerent, appartenant à la classe des verbes. Les membres de ces deux classes « connectent » automatiquement.

Dans cette conception, il faut poser que les marqueurs, éventuellement cumulés, se superposent à une relation minimale préexistante. Par les contraintes qu'ils apportent, ils spécifient cette relation minimale. Ces spécifications ne sont pas nécessairement obligatoires. Si elles sont présentes, elles précisent syntaxiquement et sémantiquement la relation; en cas d'absence, elles ne contraignent rien du tout.

Puisque les marqueurs segmentaux sont de moindre importance, il est inconcevable de matérialiser leur absence par un zéro qui serait considéré comme une réalisation phonologique nulle d'un morphème; ce dernier est tout simplement absent. Nous adhérons à cette idée. Au regard du critère de la valence passive, nous avons d'ailleurs du mal à concevoir comment un zéro pourrait contraindre la distribution de quoi que ce soit. Il faut donc poser l'analyse sans zéro de la fig. 4.

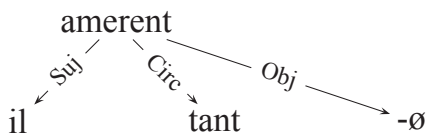

Josephé

(a) Avec zéro

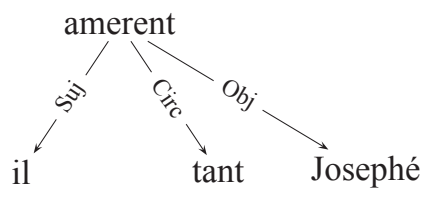

(b) Sans zéro

Fig. 4 - Analyse de il amerent tant Josephé avec et sans zéro

Par ailleurs, la présence du marqueur $-s$ n'est pas obligatoire, même pour les noms qui ont généralement une forme marquée quand ils jouent le rôle de sujet.

(7) [...] onques mes chevalier ne menja puis le tens Joseph d'Arymacie $(260,24)$

(8) onque home a nostre tens ne le pot pendre a son col a qui il n'en meschaïst $(30,31)$ 
Raisonnons par l'absurde : faut-il ajouter un zéro pour montrer qu'une marque fonctionnelle alternant avec un autre zéro n'est pas présente? Envisagée dans le cadre que nous avons choisi, il est évident que la solution offerte par les zéros n'a pas de sens pour l'afr.

\subsection{Contrainte d'adéquation marqueur/fonction}

Alain Lemaréchal ajoute un détail important à son analyse : selon lui, les marqueurs ont beau ne pas être nécessairement obligatoires il est en revanche capital qu'ils soient corrects s'ils sont présents $(1997,117)$. Ne pas satisfaire à cette contrainte d'adéquation entre la forme d'une spécification et la relation syntaxique sur laquelle elle porte génère un énoncé agrammatical et ininterprétable. Il compare les ex. 9 et 10.

(9) *Alfred chanter

(10) *Alfred chantons

Selon lui, dans l'ex. 9, la proposition reste naturellement compréhensible (même si elle n'est pas grammaticalement acceptable aux yeux de la norme) : Alfred est compatible avec chanter en vertu des parties du discours de ces deux mots. Par contre, dans l'ex. 10, la désinence -ons entre en conflit avec la troisième personne du nom propre (sauf à poser une courbe intonative adéquate). De ce fait, la phrase est tout simplement incompréhensible.

Pour ce qui concerne la déclinaison en afr., les « fautes inverses », où $-s$ apparaît au cas régime singulier, sont considérées comme très rares (Schøsler, 1984, 237-8). Toutefois, notre texte n'en est pas exempt (Marchello-Nizia et Lavrentiev, 2013, introduction); p.ex. :

(11) [...] qui Josephés avoient aporté $(259,18)$

(12) Si lesse or li contes a parler d'ax toz, et retorne a Galaad le filz Lancelot del Lac $(253,2)$

Néanmoins, ces «fautes » sont généralement limitées aux noms de personnes. Le mot filz est bien attesté sous la forme fil dans le texte, mais il tend apparemment à se figer comme un indéclinable. On peut donc raisonnablement penser que la prédiction selon laquelle un marqueur doit être correct est valable. En termes de valence passive, on peut dire que la présence d'une marque contraint fortement la distribution syntaxique de la distribution, alors que l'absence de marque ne pose aucune contrainte supplémentaire.

Ainsi, en position de sujet, les mots indéclinables (ex. 13), les féminins en - $e$, qui ne portent pas de marque opposant le cas sujet au cas régime (ex. 14) et les noms « accidentellement » non déclinés (ex. 15) reçoivent donc la même analyse syntaxique (fig. 5).

(13) pes soit a vos $(9,15)$

(14) ja mes ne passeroit damoisele par ci $(230,37)$

(15) Boort vient a son cheval $(234,37)$

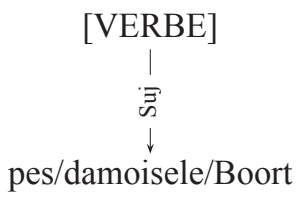

Fig. 5 - Relation entre le verbe et le sujet non marqué morphologiquement

La multiplication des paradigmes ne change strictement rien à la hiérarchie syntaxique. 


\section{Cumul des marques}

Nous allons voir comment l'approche d'Alain Lemaréchal mène à éliminer la notion de marques homonymes au profit d'une approche tenant compte des interactions entre les marques.

\subsection{Disqualification des homonymies}

Le tableau mentionné ci-dessus (tab. 1) présente une marque -s que l'on qualifie habituellement de « syncrétique », étant donné qu'elle exprime soit le cas sujet singulier, soit le cas régime pluriel. Faut-il y voir une homonymie entre deux $-s$ différents?

Alain Lemaréchal (1983) a montré que les marques se superposent et la spécification de la relation se fait de plus en plus précise au fur et à mesure que le cumul ajoute des contraintes. L'ambiguïté du marquage diminue en conséquence. Par exemple, la forme que dans les exemples qui suivent est indifférenciée, qu'il s'agisse d'une conjonction « vide » ou d'un pronom relatif :

(16) l'homme que tu vois

(17) je veux que tu viennes

La distinction traditionnelle entre deux que repose sur les compatibilités entre les parties du discours : la combinaison de ce mot avec un nom ou avec un verbe permettrait de distinguer deux types de mots. Toutefois, selon l'approche d'Alain Lemaréchal (Lemaréchal, 1997), c'est faire fausse route que de voir ici deux que distincts : il s'agit de la même forme, qui s'insère dans une combinaison de marques différentes. Seul le contexte change.

\subsection{Application à l'ancien français}

Si l'on accepte que la marque $-s$ n'est pas syncrétique, mais simplement sous-spécifiée, on n'aura aucun mal à accepter que la distribution syntaxique qu'elle impose est hétérogène et que d'autres marques, plus spécifiques, peuvent s'y superposer, comme c'est le cas de l'article défini.

Quoique facultatif ${ }^{12}$, le déterminant, et en particulier l'article défini, est souvent un indice plus fiable pour déterminer la fonction du syntagme dans lequel il se trouve. certaines formes de l'article défini sont extrêmement spécifiques : pour les noms masculins, $l i$ correspond au cas sujet, le au cas régime singulier et les au cas régime pluriel. Étant donné le critère de la valence passive, ce déterminisme morphologique correspond à l'orientation de la dépendance li/le/les $\rightarrow$ [nom]. En outre, étant donné que $-s$ est moins spécifique, le cadre formel choisi mène à le placer dans une position inférieure à l'article dans l'arbre de dépendances lorsque les deux marques se superposent. Néanmoins, la distribution moins spécifique de ce $-s$ n'en est pas anarchique pour autant, si bien qu'il ne peut apparaître si l'article en présence n'est pas compatible (ainsi, le est incompatible avec $-s$ ). La désinence reste donc bien le gouverneur du nom auquel elle s'attache.

S'il est question de formaliser les relations entre les morphèmes, le découpage et la hiérarchisation des formants ne sont pas évidents à ce stade. Si l'on découpe $l i$ en $l-+-i$, en vertu de formes comme le, et les d'une part, et $m i$ ou si d'autre part, il est facile de poser $-i \rightarrow l-$, mais de quoi dépendrait le $-s$ de chevaliers? Quelle analyse conviendrait à l'ex. 2 entre les deux de la fig. 6 ? 


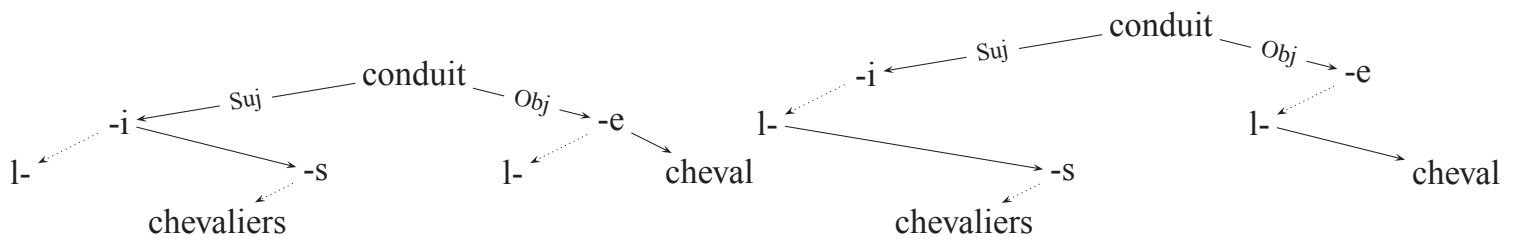

Fig. 6 - Analyses de li chevaliers conduit le cheval

Le nom dépend-il du lexème ou de la désinence de l'article ? Cette question fondamentale touche les fondations du modèle : la définition de la notion de dépendance elle-même. Pour y répondre, il ne s'agit pas simplement de disposer de critères pour déterminer l'orientation d'une dépendance, mais aussi pour repérer les dépendances entre les formes.

\section{Dépendances syntaxiques vs dépendances morphologiques}

Repérer les dépendances entre les formes implique que l'on prenne ici en considération les morphes et que soient clairement distinguées deux types de dépendances : la dépendance syntaxique (celle qui nous a occupé jusqu'ici) et la dépendance morphologique.

\subsection{Définitions de la TST}

Selon la TST, qui se refuse à procéder à une analyse syntaxique des formants du mot, l'existence d'une dépendance syntaxique entre deux formes-mots $\left(f_{l}\right.$ et $\left.f_{2}\right)$ peut être évaluée sur la base de deux critères, dits «A1 » et «A2 », qui doivent être satisfaits simultanément (2009, 25-27):

A1 : l'arrangement linéaire des formes doit être contraint dans un énoncé énonciativement neutre; p.ex. : l'article ne peut suivre le nom.

A2 : la combinaison des formes $f_{l}$ et $f_{2}$ - ou la combinaison de $f_{l}$ et de tout le sous-arbre gouverné par $f_{2}$ - doivent former une unité prosodique potentielle (ce qui est l'équivalent d'un syntagme dans le cadre de la TST); par exemple, l'article et le nom qui le suit forment un syntagme.

Une fois les associations entre deux formes découvertes, on peut appliquer les critères (dont celui de la valence passive) qui permettent de décrire l'orientation de la dépendance.

D'autre part, la TST (Mel'čuk, 2009, 12), définit la dépendance morphologique comme suit :

Une forme-mot $f_{2}$ est morphologiquement dépendante d'une forme-mot $f_{1}$ dans un énoncé si

et seulement si au moins un morphème grammatical de $f_{2}$ est sélectionné en fonction de $f_{1}$.

Par exemple, l'adjectif dépend du nom car l'accord en genre se fait en fonction d'informations relevant de la sélection du nom. Bien entendu, les dépendances syntaxiques et les dépendances morphologiques sont parfois parallèles. Par exemple, en français comme en afr., le verbe et le sujet sont unis par une dépendance syntaxique et leur accord manifeste une dépendance morphologique.

\subsection{Adaptation des définitions à l'analyse intra-mot}

Dès lors que l'on décide de découper les mots en formants et de construire un arbre de dépendances syntaxiques qui tient compte de ce niveau de syntaxe, les définitions données par la TST cessent d'être infaillibles. Bien sûr, le critère A1 reste valable (les morphèmes liés ont toujours une place fixe en morphologie grammaticale), mais qu'en est-il du critère A2 ? Dans une construction comme mit Kindern (sec. 3, 
ex. 3), mit et -n ne constituent pas un syntagme, mais le fait que - $n$ gouverne le reste du mot implique que A2 est satisfait.

Le principal problème concerne les langues où le marquage casuel est obligatoire (comme c'est le cas pour les noms latins). Les noms ne peuvent constituer un syntagme sans être pourvus d'une désinence. De ce fait, si un adjectif dépend du nom en question, comme carum dans carum amicum (ex. 18, les indices distinguent les deux morphes dans l'exposé), la dépendance amic $\rightarrow-u m_{2}$ ne satisfait pas au critère A1 car ni amic-um 2 , ni amic-carum ne forment un syntagme.

Pour résoudre ce problème sans perdre le bénéfice du critère A2, nous posons :

Soit la dépendance syntaxique intra-mot $f_{1} \rightarrow f_{2}$. A2 est satisfait si et seulement si pour route relation intra-mot $f_{2}-f_{3}$, soit $f_{1} f_{2} f_{3}$, soit $f_{1} f_{2}$ et le sous-arbre gouverné par $f_{3}$ forme une unité prosodique potentielle (= un syntagme).

En l'occurrence, si $-u m_{1} \rightarrow$ amic est une dépendance syntaxique intra-mot et $u m_{2}$ est le gouverneur de car- (fig. 7), il existe une relation entre amic- et -um 2 parce que carum amicum est une unité prosodique potentielle.

(18) Amic- - $\mathrm{um}_{1}$ car- - $\mathrm{um}_{2}$ video

ami ACC. cher ACC. je vois

« je vois (mon) cher ami »

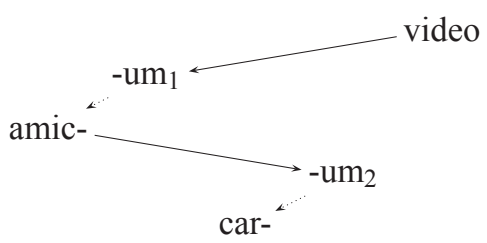

Fig. 7 - Analyse de amicum carum video

Par contre, il n'y a pas de relation syntaxique entre -um 1 et -um 2 car -um carum n'est pas un syntagme, pas plus que -um carum video n'en est un.

\subsection{Application à l'ancien français}

L'analyse d'un syntagme comme li chevaliers suit le même raisonnement : l'analyse est nécessairement la suivante (fig. 8), qui convient aux principes énoncés précédemment : - $i$ et chevaliers ne formeraient pas un syntagme. L'« accord » entre $-i$ et $-s$ ne doit donc pas être représenté.

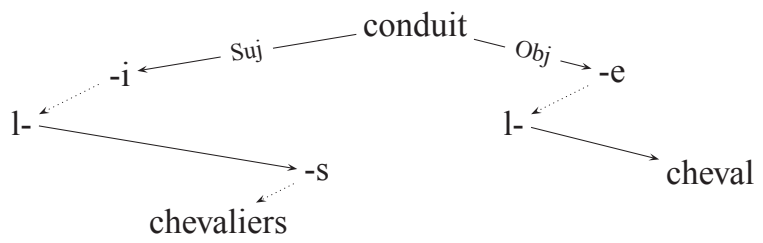

Fig. 8 - Choix de l'analyse li chevaliers conduit le cheval

Cependant, il est connu que les formes féminines de l'article ne sont pas spécifiques à la fonction syntaxique, comme le montre le tableau classique (tab. 2). 


\begin{tabular}{lllll} 
& \multicolumn{2}{c}{ MASC. } & \multicolumn{2}{c}{ FÉM. } \\
& sg. & pl. & sg. & pl. \\
\hline CAS SUJET & li & li & \multirow{2}{*}{ la } & les \\
CAS RÉGIME & le & les & & \\
Tab. 2 - Paradigme de l'article défini
\end{tabular}

Or, il y a deux cas de figure en ce qui concerne la flexion du nom féminin : soit le -s est présent et l'article lui est cumulé, soit le $-s$ est absent.

(19) la nuiz fu venue $(93,17)$

(20) la reïne vient a Galaad $(21,5)$

Au vu des relations considérées jusqu'ici, une forme comme la ne contraint en rien la distribution du syntagme : c'est la classe nominale qui détermine la distribution de la structure. Ainsi, en présence d'un - $s$ de flexion (analysé comme ci-dessus, sec. 3), l'article est nécessairement positionné plus bas dans l'arbre. D'autre part, si le $-s$ est absent, l'article n'est pas plus spécifique pour autant et la structure reste très similaire (fig. 9 ; la décomposition de l'article n'a ici aucune incidence sur la hiérarchie).
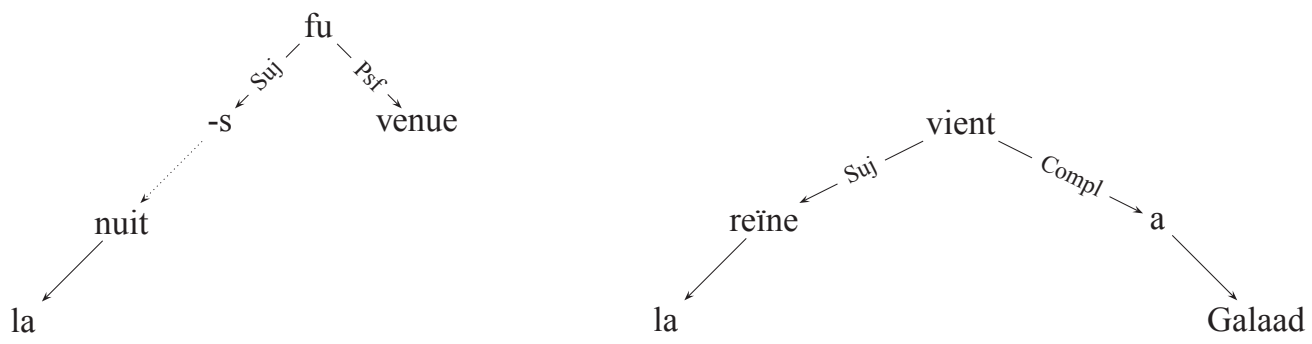

Fig. 9 - Analyse de la nuiz... et de la rë̈ne...

\section{Conclusion}

Nous avons décrit le réseau de dépendances à l'œuvre au cœur du syntagme nominal en afr. en nous appuyant sur des principes généraux :

- le critère de la valence passive;

- la décomposition en morphes;

- la notion de spécification, entendue comme un cumul de marques imposant des contraintes sur la distribution syntaxique.

L'application de ces principes à nos matériaux nous a mené à deux conclusions fortes :

1. Il est possible de décrire le comportement des unités sans recourir à des zéros. Ainsi, la marque $-s$ n'alterne pas avec $\phi$, mais s'ajoute facultativement à une relation.

2. Certaines unités qui sont généralement décrites comme des membres d'un même paradigme morphologique ont un impact différent sur la spécification des relations syntaxiques. En conséquence, elles sont situées à des niveaux différents dans la hiérarchie syntaxique. Ainsi, l'article li est beaucoup plus contraignant que l'article $l a$, sous-spécifié. Entre eux deux, seul $l i$ contraint la distribution du syntagme.

Cela ne signifie pas que les hiérarchies proposées dans ce travail sont définitives : l'application des mêmes critères à l'ensemble des marques devrait mener à une réanalyse des cas examinés ci-dessus. Nous pensons 
toutefois que la démarche présente un intérêt non négligeable pour la description de la langue ancienne (et des langues en général) : elle invite à reconsidérer la notion de paradigme morphologique pour lui donner une dimension plus syntaxique, plus hiérarchique.

La nature de nos matériaux et de nos choix théoriques nous ont également obligé à critiquer et à revoir les principes généraux proposés par la TST pour identifier les dépendance. Ainsi, la définition du critère dit «A2 », selon lequel deux termes unis par une dépendance doivent former un syntagme, a dû être précisée pour que l'analyse tenant compte des morphes reste adéquatement cadrée par des principes formels applicables. Voilà un échange dont nous nous réjouissons.

\section{Références bibliographiques}

Bloomfield, Leonard (1933). Language, London : George Allen \& Undwin Ltd.

Buridant, Claude (2000). Grammaire nouvelle de l'ancien français, Paris : Sedes.

Chambon, Jean-Pierre (2003). « La déclinaison en ancien occitan, ou : comment s'en débarrasser? Une réanalyse non orthodoxe de la flexion substantivale », dans Revue de linguistique romane 67 : 342-363.

Chambon, Jean-Pierre et Davidsdottir, Rosa (2007). « Approche de la déclinaison des substantifs en ancien français : de Moignet à Skårup (lecture critique et suggestions) », dans Bulletin de la Société de Linguistique de Paris 102(1) : 173-192.

Detges, Ulrich (2009). « How useful is case morphology? The loss of the Old French two-case system within a theory of Preferred Argument Structure », dans Barđdal, Jóhanna et Shobhana, Chelliah, éds, The Role of Semantic, Pragmatic, and Discourse Factors in the Development of Case, Amsterdam : Benjamins (Studies in Language Companion Series 108) : $93-120$

Garde, Paul (1981). " Des parties du discours, notamment en russe », dans Bulletin de la Société de Linguistique de Paris 76(1) : 155-189.

Groß, Thomas (2011). « Catenae in Morphology », dans Gerdes, Kim, Hajičová, Elena et Wanner, Leo, éds, Proceedings of Depling 2011, International Conference on Dependency Linguistics, Barcelona, Barcelona : 47-57, http://depling.org/proceedingsDepling2011/.

Haegemann, Lilinane (21994). Introduction to Government and Binding Theory, Oxford and Malden : Blackwell.

Lazard, Gilbert (1984). «La distinction entre nom et verbe en syntaxe et en morphologie », dans Modèles linguistiques $6(1): 29-39$

Lemaréchal, Alain (1983). "Sur la prétendue homonymie des marques de fonction : la superposition des marques », dans Bulletin de la Société de Linguistique de Paris 78(1) : 53-76.

Lemaréchal, Alain (1997). Zéro(s), Paris : Presses universitaires de France (Linguistique nouvelle).

Marchello-Nizia, Christiane et Lavrentiev, Alexei, éds (2013). Queste del saint Graal. Édition numérique interactive du manuscrit de Lyon (Bibliothèque municipale, P.A. 77), http://portal. textometrie .org/txm/, juillet 2013.

Mazziotta, Nicolas (2013). «Grammatical markers and grammatical relations in the simple clause in Old French », dans Gerdes, Kim, Hajičová, Elena et Wanner, Leo, éds, Proceedings of Depling 2013, Second International Conference on Dependency Linguistics, Prague, Barcelona : 207-216, http://depling .org/proceedingsDepling2013/.

Mel'čuk, Igor (2009). « Dependency in natural language », dans Polguère, Alain et Mel'čuk, Igor, éds, Dependency in linguistic description, Amsterdam and Philadelphia : John Benjamins : 1-110.

Moignet, Gérard (21988). Grammaire de l'ancien français. Morphologie - Syntaxe, Paris : Klincksieck (Initiation à la linguistique. Série B. Problèmes et méthodes 2). 
Prévost, Sophie et Stein, Achim, éds (2013). Syntactic Reference Corpus of Medieval French, Lyon/Stuttgart : ENS de Lyon; Lattice, Paris; Universität Stuttgart, http://srcmf . org.

Schøsler, Lene (1984). La déclinaison bicasuelle de l'ancien français : son rôle dans la syntaxe de la phrase, les causes de sa disparation, Odense : Odense University Press (Etudes romanes de l’Université d'Odense 19).

Schøsler, Lene (2013). « The development of the declension system », dans Arteaga, Deborah L., éd., Research on Old French : the state of the art, London : Springer : 167-186.

Stein, Peter et Benneckenstein, Claudia (2006). «Historische Fallstudie : Altfranzösisch », dans Àgel, Vilmos, Eichiner, Ludwig M., Eroms, Hans-Werner, Hellwig, Peter, Heringer, Hans Jürgen et Lobin, Henning, éds, Dependenz und Valenz. Ein internationales Handbuch der zeitgenössischen Forschung. 2. Halbband, Berlin and New York : Walter de Gruyter (Handbücher zur Sprach- und Kommunikationswissenschaft) : 1508-1515.

Tesnière, Lucien (2 1966). Éléments de syntaxe structurale, Paris : Klincksieck.

Zwicky, Arnold M. (1985). « Heads », dans Journal of linguistics 21 : 1-29.

\section{Notes}

${ }^{1}$ Cette contribution est une version étendue, adaptée et traduite de la communication que nous avons présentée en anglais au colloque Depling 2013 (Mazziotta, 2013). Nous voudrions remercier les personnes suivantes pour leurs remarques et leur aide (l'ordre est indifférent) : Lene Schøsler, Thomas Groß, Sylvain Kahane, Timothy Osborne.

${ }^{2}$ Sauf indication contraire, les termes dépendance, gouverneur, dépendant, actant, etc., ainsi que le symbole « $\rightarrow$ »ntre un gouverneur et un dépendant sont ici à situer dans le cadre de la structuration syntaxique de surface (Mel’čuk, 2009, 6-7).

${ }^{3}$ Il faut ici mentionner les travaux de Lene Schøsler qui, depuis Schøsler 1984, emploie l'approche de Lucien Tesnière (1966) avec fruit pour décrire les relations actancielles.

${ }^{4}$ Sauf indication spécifique, les termes valence et valence passive ne sont pas ici strictement réservés au verbe et à ses dépendants. Ces notions s'appliquent à toute relation de subordination (comme celle qui unit le nom et l'adjectif épithète).

${ }^{5}$ Il ne s'agit pas ici de traiter l'ensemble du texte et de livrer une analyse statistique des phénomènes observés. Ce travail, qui nécessite une annotation morphologique spécifique, est en cours de réalisation par nos soins. (ibid.)

${ }^{6} \mathrm{Ce}$ critère n'est pas infaillible et d'autres critères (morphologiques et sémantiques) peuvent être mobilisés s'il est insuffisant

${ }^{7}$ S'il est vrai que le mot bel peut en effet être supprimé sans réduire la grammaticalité de la construction, le critère de la valence passive ne doit pas être confondu avec la possibilité d'omettre une unité. Selon la TST, les gouverneurs ne peuvent généralement pas être omis, mais il y a des exceptions ; p.ex, en anglais, la conjonction de subordination that contraint la distribution de la complétive et gouverne donc le verbe subordonné, mais elle peut toutefois être omise (Mel'čuk, 2009, 42).

${ }^{8}$ On notera l'emploi des capitales pour représenter les lexèmes (unités abstraites), qui est de moindre importance pour l'argumentation.

${ }^{9}$ Cette approche, on le verra, nous mènera à revoir les définitions fondamentales de la TST (sec. 6).

${ }^{10}$ Nous nous concentrerons ici exclusivement sur les morphes flexionnels, laissant de côté la morphologie dérivationnelle.

${ }^{11}$ L'arbre représente également l'ordre des unités. Cet aspect ne concerne pas cette étude, mais nous le conservons pour des raisons ergonomiques.

${ }^{12}$ Tous les noms peuvent être employés sans déterminant, ce qui implique que le syntagme exprime une valeur générale ou une classe référentielle prise dans son ensemble (Moignet, 1988, 105-11). 